\title{
Kinetics of occupancy of defect states in poly(3- hexylthiophene):fullerene solar cells
}

Pablo P. Boix ${ }^{1}$, Jon Ajuria $^{2}$, Ikerne Etxebarria ${ }^{2}$ Roberto Pacios $^{2}$, and Germà Garcia-Belmonte ${ }^{1 *}$

1 Photovoltaic and Optoelectronic Devices Group, Departament de Física, Universitat Jaume I, ES-12071 Castelló, Spain

2 Department of Microsystems, IKERLAN-IK4 S. Coop. Goiru Kalea 9. Polo Innovación Garaia. ES- 20500 Arrasate-Mondragón, Gipuzkoa, Spain

\begin{abstract}
Energetics and kinetics of defects in the effective band gap of organic bulk heterojunctions are determined by means of capacitance methods. The technique consists of calculating the junction capacitance derivative with respect to the angular frequency of the small voltage perturbation applied to thin film poly(3-hexylthiophene) (P3HT) and [6,6]-phenyl $\mathrm{C}_{61}$-butyric acid methyl ester solar cells, varying the temperature. The analysis reveals the presence of defect bands (shallow acceptors) centered at $E_{0}=35 \mathrm{meV}$ above the highest-occupied molecular orbital level of P3HT. The total density of defects results of order $10^{16} \mathrm{~cm}^{-3}$. Characteristic frequency is obtained to be situated within the range of 1-10 Hz. Defect bands acting as negatively charged levels are responsible for the $p$-doping of the active layer and the band-bending near the cathode contact, as derived from Mott-Schottky capacitance-voltage analysis.
\end{abstract}

Keywords: Organic solar cells, defects, cathode, capacitance, Mott-Schottky.

*Corresponding author: G. Garcia-Belmonte, e-mail: garciag@uji.es, tel.: +34 964 387548, fax: +34964 729218 


\section{Introduction.}

Conducting organic compounds are at the heart of bulk heterojunction solar cells, formed by an interpenetrating blend of an optically active polymer and electron accepting molecules, and constitute a very promising route towards cheap and versatile solar cells. The combination of P3HT and $[6,6]$-phenyl $\mathrm{C}_{61}$-butyric acid methyl ester (PCBM) in organic blends has shown high photovoltaic performance [1]. Solutionprocessed polythiophene:fullerene cells achieved over $6 \%$ efficiency by the use of recently studied materials as well as additives optimizing the phase separation [2]. In order to further improve organic solar cell performance extracting information about defect states and their role as trapping or recombination centers seems to be crucial. It is well known that P3HT is a conjugated polymer that in exposure to oxygen [3], or moisture [4] results $p$-doped exhibiting relatively high levels of free carries $\left(10^{15}-10^{17}\right.$ $\mathrm{cm}^{-3}$ ) [5]. Under such conditions, it has been suggested that the P3HT-metal contact forms a Schottky barrier behavior: band bending with a corresponding majority-carrier depletion zone made up at the cathode contact [6,7], due to the presence of acceptor defects (see Fig. 1). In Fig. 1 only relevant energy levels for transport and energetics are drawn because of the effective medium formed by the acceptor-donor blend. This band structure across the active layer differs from that expected using undoped materials, in which the potential drops linearly between the contacts [8]. Impedance and capacitance measurements have been used to evidence such band bending $[9,10]$ usually exhibiting a Mott-Schottky characteristics ( $C^{-2} \propto V$ ), which can be readily interpreted as a strong indication of the presence of negatively charged defects within the P3HT energy gap. Alternative techniques like Kelvin probe[11], and ultraviolet photoelectron spectroscopy [12] have given further evidence of the formation of depletion zones at metal/organic contacts with the corresponding band bending caused by the presence of impurity levels.

Information about bulk defect states in polymer-fullerene solar cells has been extracted from subgap optical absorption experiments [13], low excitation intensity transient absorption spectroscopy [14], and thermally stimulated current techniques [15, 16]. It is widely recognized that defect states and traps might have important effects on the solar cells performance [17]. For instance, charged defects within the gap alter the electrical field profile. The negative impact of oxygen-induced traps on the charge transport in poly(3-hexylthiophene) diodes has been recently addressed [16, 18].

This work investigates the density, energetic, and kinetics of defect states within the gap of P3HT, following our previous results [9]. The study is performed by analyzing 
capacitance dependences on both bias voltage $C(V)$ and frequency $C(f)$ of complete cells, following known techniques for characterizing inorganic semiconductor contact effects [19-21]. It is worth noting here that organic semiconductors exhibit strong charge localization features in comparison to their inorganic counterparts. Inherent energetic disorder of organic semiconductors resulting from structural inhomogeneities or chemical impurities enhances charge localization and gives rise to carrier trapping. Negatively charged defects (acceptors states) donate a hole to the transport-related highest-occupied molecular orbital (HOMO) levels and are then responsible for the $p$ doping of the polymer. Although the techniques employed in this study were devised to investigate ionized impurity levels in inorganic semiconductors, charged defects in conjugated polymers act in a similar way in terms of macroscopic electrostatics.

\section{Experimental section}

Solar cells of structure indium tin oxide (ITO)/poly(ethylenethioxythiophene):poly(styrenesulfonic) acid (PEDOT:PSS)/P3HT:PCBM/Ca/Ag were built according to the following procedure: In-house structured ITO substrates of $4 \Omega / \square$ were cleaned ultrasonic baths of acetone, isopropanol and clean isopropanol during 5 minutes. Substrates were then ozone treated for 5 minutes. Clevios P Al4083 was spin-coated in air onto the ITO to produce $40 \mathrm{~nm}$ of PEDOT:PSS. Films were annealed at $100^{\circ} \mathrm{C}$ for $5 \mathrm{~min}$ to remove any possible residual water. The solutions containing weight ratio (1:0.8) of P3HT (from Rieke Materials) and PCBM (from Nano-C) in chlorobenzene were prepared in ambient conditions at room temperature. Solutions were then let to stir overnight in air at $50^{\circ} \mathrm{C}$. After cooling to room temperature, solutions were spin-coated at 2000-2500 rpm to achieve thickness of 100-120 nm measured by profilometric techniques. The resulting films were then placed into a nitrogen glove-box where calcium and silver were thermally evaporated through a metal shadow under high vaccuum $\left(\sim 4 \times 10^{-4} \mathrm{~Pa}\right)$ to produce $\mathrm{Ca}(10 \mathrm{~nm}) / \operatorname{Ag}(200 \mathrm{~nm})$ upper electrodes. Finally, all substrates were encapsulated with an epoxy resin and glass, taking special care to leave at least $2 \mathrm{~mm}$ from the diode to the encapsulating glass to prevent any oxygen/moisture penetration from the edges. The epoxy resin (ELC 2500 clear) was cured with a $365 \mathrm{~nm}$ UV light of $90 \mathrm{~mW} \mathrm{~cm}{ }^{-2}$ during 30 seconds. The resulting devices were then thermally annealed in nitrogen at $140{ }^{\circ} \mathrm{C}$ for $15 \mathrm{~min}$ and taken to air for characterization. This procedure has demonstrated stability in samples kept in standard lab conditions (no intense light and 
ambient temperature around $22^{\circ} \mathrm{C}$ ) for longer than a year. The capacitance measuring method was described in the previous work [9]. Capacitance spectra were measured at decreased temperatures starting at $315 \mathrm{~K}$. A final spectrum was again registered at room-temperature to check measurement reproducibility. Photovoltaic response was observed to result in conversion efficiencies around 3.5\% [9]. Current density-voltage $(J-V)$ characteristics of devices were taken using a Keithley 4200 source unit under 1 sun AM1.5G illumination conditions from a xenon lamp based Oriel $9600150 \mathrm{~W}$ Sun Simulator. The calibration of the incident light for $J-V$ measurements was performed calibrated silicon photodiode, making sure that short-circuit current $J_{\text {sc }}$ measured under the calibrated $1000 \mathrm{~W} \mathrm{~m}^{-2}$ white incident light coincides with $J_{\mathrm{sc}}$ calculated by integrating the external quantum efficiency times the photon flux across the visible spectrum. In this way, we can avoid any type of mistake when reporting efficiencies.

\section{Results and discussion}

Defect states within the bandgap contribute to the junction capacitance depending on their energy and space position. Only defects below (in case of a p-doped semiconductor) certain demarcation energy $E_{\omega}=k_{\mathrm{B}} T \ln \left(\omega_{0} / \omega\right)$ will be able to contribute to the junction capacitance $[22,23]$. Here $k_{\mathrm{B}} T$ corresponds to the thermal energy and $\omega_{0}$ denotes a characteristic frequency, and $\omega=2 \pi f$ the angular frequency of the perturbation. The capacitance spectra is viewed as an energy spectroscopy which explores energy levels of faster states at high frequencies (near the HOMO of the polymer), and sweeps towards mid-gap as the frequency decreases (slower defects far from the HOMO level), i.e. $E_{\omega}=E-E_{\mathrm{HOMO}}$ (Fig. 1). The density of defect states (DOS) $g_{t}(E)$ was stated to be related to the derivative of the capacitance with respect to the frequency as [22],

$$
g_{t}\left(E_{\omega}\right)=-\frac{V_{\mathrm{fb}}}{q w k_{\mathrm{B}} T} \frac{d C(\omega)}{d \ln \omega}
$$

being $w$ the width of the depletion zone, and $V_{\mathrm{fb}}$ is the flat-band potential, which corresponds to the energy shift between the effective cathode workfunction and the Fermi level located near the donor HOMO by effect of $p$-doping (Fig. 1). Equation (1) has been recently used within the field of organic electronics [24, 25]. Although we are assuming in this study a bulk origin for the defect band, the response of interfacial states at the contacts cannot be completely discarded.

We show in Fig. 2(a) typical capacitance spectra $C(f)$ obtained at zero bias in the 
dark at different temperatures. $C(f)$ curves exhibit an increment of the capacitance towards low frequencies $(0.1-10 \mathrm{~Hz})$ with respect to the geometrical value $C_{g} \approx 3 \mathrm{nF}$, resulting from contributions of defect levels in the gap. We recently demonstrated that such defects belong to the polymer in analyzing P3HT diodes along with P3HT:PCBM solar cells [9]. Because a complete step is observed in $C(f)$, the inflection point serves to define a frequency $f_{\mathrm{m}}$ related to the defect band kinetic response, which corresponds to the minimum of the frequency derivative $d C(f) / d \ln f$ [arrows in Fig. 2(b)]. The frequency $f_{\mathrm{m}}$ exhibits an Arrhenius behavior $f_{\mathrm{m}}=f_{0} \exp \left(-E_{0} / k_{\mathrm{B}} T\right)$ as shown in Fig. 2(c), from which an activation energy $E_{0}=35 \mathrm{meV}$ and frequency prefactor $\omega_{0}=2 \pi f_{0}=210 \mathrm{~s}^{-1}$ are obtained.

Mott-Schottky characteristics is displayed in Fig. 3 from the capacitance measured at room temperature and low frequency $(100 \mathrm{~Hz})$ as $C^{-2}(V)$. Such response is a clear indication of the Schottky-barrier formation between organic blend and the cathode metal (Fig. 1). The intercept with the real axis is related to the flat-band potential $V_{\mathrm{fb}}=0.35 \mathrm{~V}$, which corresponds to the energy offset between the blend Femi level and the Ca work function. The blend Fermi level approaches the polymer HOMO because of the $p$-doping. The effective metal work function might be altered by the presence of a thin oxide layer produced by the metal oxidation (dipole layer). These two facts give rise to the $V_{\mathrm{fb}}$ value actually measured, and employed in the calculations using Eq. (1). Full-depletion is observed at $V<0$ V. For voltages $V<V_{\mathrm{fb}}$ the depletion zone (which confines the electrical field) width is modulated. An estimation of the density of dopants $n \approx 9 \times 10^{15} \mathrm{~cm}^{-3}$ responsible for the $p$-doping is derived from the slope of the linear part in Fig. 3 as explained in ref. [9]. By applying Eq. (1) to the capacitance spectra in Fig. 2(a), the DOS obtained as a function of $E-E_{\text {HOMO }}$ is plotted in Fig. 4. Here we assume that the active layer is fully depleted at zero bias, so that the depletion width can be approximated by the blend thickness. The application of Eq. (1) is wholly justified at zero bias because the band bending is negligible.

The DOS calculated can be analyzed by regarding either a Gaussian or doubleexponential shape centered at the maximum of the distribution $E_{0} \approx 35 \mathrm{meV}$ (independently extracted from the temperature analysis). The disorder parameter $\sigma$, which accounts for the broadening of the DOS and calculated from the half width, is approximately equal to $30 \mathrm{meV}$. Finally, the density of defects $n_{t}$, calculated by integration of the DOS, results approximately equal to $5 \times 10^{15} \mathrm{~cm}^{-3}$. By examining Fig. 4 one can infer that the DOS appears to be slightly temperature-dependent, exhibiting a 
two-fold reduction in $n_{t}$ within the employed temperature range.

The value obtained for the activation energy indicates that the defect band is also formed by shallow electron acceptors. It should be noted here that we distinguish between defect states responsible for the $p$-doping (extracted from the $C^{-2}(V)$ analysis at $100 \mathrm{~Hz}$ ) from much slower traps (responding below $10 \mathrm{~Hz}$ ). The factor which allows distinguishing between dopants or traps is then the difference in response kinetics. In both cases the origin of the defect states reported here must be found within intrinsic acceptor levels induced during film preparation. One possible source was proposed by Gregg [26] as morphological defects related to stacking faults or distorted covalent bonds resulting from the twisting of a planar conjugated polymer backbone. Other defects bands might appear as tails of localized states near HOMO and lowestunoccupied molecular orbital levels, increasing as the disorder increases. Shallow traps centered at 50 and $105 \mathrm{meV}$ were previously indentified using thermally-stimulated current techniques on polymer (P3HT) diodes [16]. Activation energies within the range of 24-34 meV were also encountered by means of capacitance tools for the system ITO/ poly(2-methoxy-5-(3'-7'-dimethyloctyloxy)-1,4-phenylenevinylene):PCBM/A1 [27].

The frequency exponential prefactor encountered $\omega_{0}=210 \mathrm{~s}^{-1}$ results very low in comparison to the values expected from molecular vibration of phonon modes $\left(\omega_{0}=10^{13}-10^{12} \mathrm{~s}^{-1}\right)$, which were used in our previous analysis [9], or extracted by others from admittance methods applied on organic solar cells $\left(\omega_{0}=10^{7}-10^{9} \mathrm{~s}^{-1}\right)[27$, 28]. In our previous study [9] the estimation of the defect band energy ( $E_{0} \approx 380 \mathrm{meV}$ ) was overestimated because of the assumed high value for $\omega_{0}$. The defect density results within the same order of magnitude. We stress here that an exact determination of $\omega_{0}$ is then extremely important because it establishes the proper energy range of the defect band level. For inorganic semiconductors the characteristic frequency is usually related to the kinetics of occupation of the defect state as $\omega_{0}=2 \beta N_{p}$ [29]. Here $\beta$ accounts for a capture coefficient and $N_{p}$ to the density of valence band states. Assuming $N_{p}=10^{20} \mathrm{~cm}^{-3}$ from typical monomer density and $\omega_{0}=210 \mathrm{~s}^{-1}$ from Fig. 2(c), it is inferred that $\beta \approx 10^{-18} \mathrm{~cm}^{3} \mathrm{~s}^{-1}$, again a very low value for a capture coefficient. In case of organic semiconductors usual interpretations given to the capture coefficient $\beta$ should be linked to microscopic mechanisms accounting for the charge transfer reaction responsible for the defect charging. Usually the Marcus theory of charge transfer is employed to account for defect charging and trapping (this is a distinctive feature of organic semiconductors in comparison with their inorganic counterparts) [30]. The 
charge transfer rate $k$ in the semiclassical expression is written as

$$
k=\frac{2 \pi}{h}\left|V_{i f}\right|^{2} \sqrt{\frac{1}{4 \pi \lambda k_{\mathrm{B}} T}} \exp \left(-\frac{\left(\Delta G_{0}+\lambda\right)^{2}}{4 \lambda k_{\mathrm{B}} T}\right)
$$

where $\lambda$ is the reorganization energy induced by the electron transfer, $\Delta G_{0}$ is the variation of the Gibbs free energy during the reaction, and $V_{\text {if }}$ corresponds to the electronic coupling matrix element. By identifying terms one can obtain that $\omega_{0}=2 \pi / h\left|V_{i f}\right|^{2}\left(4 \pi \lambda k_{\mathrm{B}} T\right)^{-1 / 2}$. The frequency prefactor is then a function of the coupling matrix of the charge transfer, which basically establishes the time scale of the reaction. Charge transfer reactions in organic compounds may be so slow as to explain the observed characteristic frequency of order $10^{2} \mathrm{~s}^{-1}$. There exist some examples of slow charge-transfer kinetics: minutes scale self-trapping processes were observed in polycrystalline pentacene by time-resolved electric force microscopy [31], or slow localization dynamics of electrons on ice surfaces [32]. Therefore molecular and lattice distortions in presence of a charge carrier would lie behind the observed retardation effects.

\section{Conclusions}

We then conclude that the defect bands found in this study have a great influence on the electrical field profile when charged because of the defect DOS of order $10^{16} \mathrm{~cm}^{-3}$. Such defect band is responsible for the $p$-doping of the active layer as stated from the Mott-Schottky capacitance-voltage analysis. At usual operating potentials close to the maximum power point, majority carriers shield the electrical field outside the depletion zone. Device physics modeling must include such defect bands on simulation tools.

\section{Acknowledgements}

We thank financial support from Ministerio de Educacion y Ciencia under project HOPE CSD2007-00007 (Consolider-Ingenio 2010), and Universitat Jaume I (P1.1B2008-32). Use of SCIC (Universitat Jaume I) instrumental facilities is acknowledged. 


\section{References}

[1] W. Ma, C. Yang, X. Gong, K.-S. Lee, A.J. Heeger, Adv. Funct. Mater. 15 (2005) 1617.

[2] G. Zhao, Y. Hi, Y. Li, Adv. Mater. 22 (2010) 4355.

[3] M.S.A. Abdou, F.P. Orfino, Y. Son, S. Holdcroft, J. Am. Chem. Soc. 119 (1997) 4518.

[4] S. Hoshino, M. Yoshida, S. Uemura, T. Kodzasa, N. Takada, T. Kamata, K. Yase, J. Appl. Phys. 95 (2004) 5088.

[5] Z. Liang, A. Nardes, D. Wang, J.J. Berry, B.A. Gregg, Chem. Mater. 21 (2009) 4914.

[6] G. Dennler, C. Lungenschmied, N.S. Saricifti, R. Schwödiauer, S. Bauer, H. Reiss, Appl. Phys. Lett. 87 (2005) 163501.

[7] G. Garcia-Belmonte, A. Munar, E.M. Barea, J. Bisquert, I. Ugarte, R. Pacios, Org. Electron. 9 (2008) 847.

[8] J. Bisquert, G. Garcia-Belmonte, J. Phys. Chem. Lett. 2 (2011) 1950.

[9] P.P. Boix, G. Garcia-Belmonte, U. Muñecas, M. Neophytou, C. Waldauf, R. Pacios, Appl. Phys. Lett. 95 (2009) 233302.

[10] G. Garcia-Belmonte, P.P. Boix, J. Bisquert, M. Sessolo, H.J. Bolink, Sol. Energy Mater. Sol. Cells 94 (2010) 366.

[11] H. Ishii, N. Hayashi, E. Ito, Y. Washizu, K. Sugi, Y. Kimura, M. Niwano, Y. Ouchi, K. Seki, Phys. Status Solidi A 201 (2004) 1075.

[12] T. Nishi, K. Kanai, Y. Ouchi, M.R. Willis, K. Seki, Chem. Phys. Lett. 414 (2005) 479.

[13] L. Goris, A. Poruba, L. Hod'ákova, M. Vanecek, K. Haenen, M. Nesládek, P. Wagner, D. Vanderzande, L. De Schepper, J.V. Manca, Appl. Phys. Lett. 88 (2006) 052113.

[14] A.F. Nogueira, I. Montanari, J. Nelson, J.R. Durrant, C. Winder, Sariciftci, C. Brabec, J. Phys. Chem. B 107 (2003) 1567.

[15] T.P. Nguyen, Phys. Stat. Sol. (a) 205 (2008) 162.

[16] J. Schafferhans, A. Baumann, C. Deibel, V. Dyakonov, Appl. Phys. Lett. 93 (2008) 093303.

[17] L.G. Kaake, P.F. Barbara, X.-Y. Zhu, J. Phys. Chem. Lett. 1 (2010) 628. 
[18] J. Schafferhans, A. Baumann, A. Wagenpfahl, C. Deibel, V. Dyakonov, Org. Electron. 11 (2010) 1693.

[19] L.C. Kimerling, J. Appl. Phys. 45 (1974) 1839.

[20] I. Balberg, J. Appl. Phys. 58 (1985) 2603.

[21] S.S. Hegedus, W.N. Shafarman, Prog. Photovoltaics 12 (2004) 155.

[22] T. Walter, R. Herberholz, C. Müller, H.W. Schock, J. Appl. Phys. 80 (1996) 4411.

[23] S.S. Hegedus, E.A. Fagen, J. Appl. Phys. 71 (1992) 5941.

[24] P. Yu, D. Mencaraglia, A. Darga, A. Migan, R. Rabdbeh, B. Ratier, A. Moliton, Phys. Status Solidi C 3-4 (2010) 1000.

[25] K.S. Nalwa, R.C. Mahadevapuram, S. Chaudhary, Appl. Phys. Lett. 98 (2011) 093306.

[26] B.A. Gregg, J. Phys. Chem. C 113 (2009) 5899.

[27] V. Dyakonov, D. Godovsky, J. Meyer, J. Parisi, C.J. Brabec, N.S. Sariciftci, J.C. Hummelen, Synthetic Met. 124 (2001) 103.

[28] V. Dyakonov, I. Riedel, Z. Chiguvare, C. Deibel, J. Parisi, C. Brabec, N.S. Sariciftci, J.C. Hummelen, Materials Research Society Symposium Proceedings 275 (2002) P7.10.1.

[29] J.M. Montero, J. Bisquert, G. Garcia-Belmonte, E.M. Barea, H.J. Bolink, Org. Electron. 10 (2009) 305.

[30] R.A. Marcus, Rev. Mod. Phys. 65/3 (1993) 599.

[31] M. Jaquith, E.M. Muller, J.A. Marohn, J. Phys. Chem. B 111 (2007) 7711.

[32] U. Bovensiepen, C. Gahl, J. Stähler, M. Bockstedte, M. Meyer, F. Baletto, S. Scandolo, X.-Y. Zhu, A. Rubio, M. Wolf, J. Phys. Chem. C 113 (2009) 979. 


\section{Figure captions}

\section{Fig. 1}

Schematic representation of the band diagram of a $p$-doped donor polymer-acceptor fullerene blend at zero bias voltage. A depletion zone is formed near the cathode contact, and an ohmic contact at the hole transporting layer (HTL) interface. We show a case exhibiting not full depletion. The crossing point between the Fermi level $E_{F}$ and the defect energy $E_{t}$ within the depletion zone marks the position of occupancy change in equilibrium. $E_{\omega 1}$ corresponds to a measurement condition at which defects cannot respond to the perturbation because of the high frequency or low temperature. Instead $E_{\omega 2}$ (low frequency or high temperature conditions) is far above the defect energy so that the occupancy is determined by $E_{F}$.

\section{Fig. 2}

(a) Capacitance spectra measured at zero bias voltage at different temperatures of P3HT:PCBM solar cells with weight ratio (1:0.8). (b) Low-frequency part of the derivative $d C(f) / d \ln f$ of capacitance spectra indicating the position of the characteristics frequency $f_{\mathrm{m}}$ (arrows). (c) Arrhenius plot of the characteristics frequency.

\section{Fig. 3}

Mott-Schottky characteristics $\left[C^{-2}(V)\right]$ of the cell of Fig. 2 exhibiting full depletion at reverse bias and a linear relationship at low forward bias $(0.1-0.3 \mathrm{~V})$.

\section{Fig. 4}

Density of defect states as a function of the energy with respect to the P3HT HOMO level (demarcation energy), $E-E_{\mathrm{HOMO}}$, at different temperatures calculated using Eq. (1) and the capacitance spectra in Fig. 2(a). 